\title{
CRMP5 regulates cell proliferation and development of colorectal cancer via MAPK-dependent signaling
}

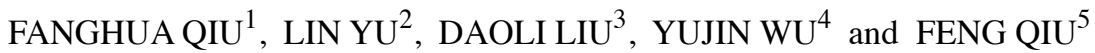 \\ ${ }^{1}$ Department of Infection Control, Guangzhou Hospital of Traditional Chinese Medicine, Guangzhou, \\ Guangdong 510130; ${ }^{2}$ Department of Clinical Laboratory, The First Affiliated Hospital of Guangzhou Medical \\ University, Guangzhou 510120; Departments of ${ }^{3}$ Clinical Laboratory and ${ }^{4}$ Spleen and Gastroenterology, Guangzhou \\ Hospital of Traditional Chinese Medicine, Guangzhou, Guangdong 510130; ${ }^{5}$ Department of Laboratory Medicine, \\ Nanhai Hospital, Southern Medical University, Foshan, Guangdong, 528244, P.R. China
}

Received December 17, 2019; Accepted April 12, 2021

DOI: 10.3892/ol.2021.13008

\begin{abstract}
Colorectal cancer (CRC) is one of the most common malignancies worldwide. Collapsin response mediator protein 5 (CRMP5) belongs to a family of five cytosolic proteins that serve a major role in neural development. CRMP5 has been identified as a biomarker of numerous cancer types, including lung cancer and glioblastoma. However, the role of CRMP5 in CRC remains unclear. In the present study, CRMP5 was characterized as a novel biomarker of poor survival in CRC. CRMP5-overexpression in CRC cells promoted cell proliferation and migration while CPMP5-knockdown decreased cell growth and migration. A novel mechanism was uncovered, by which CRMP5 regulates MAPK signaling to drive CRC cell proliferation and development. Furthermore, CRMP5-overexpression induced chemotherapy resistance and tumor recurrence in CRC. Taken together, these results demonstrated the important role of CRMP5 in the development and proliferation of CRC cells and suggested that CRMP5 may be a novel therapeutic target for CRC.
\end{abstract}

\section{Introduction}

Colorectal cancer (CRC) is the development of cancer from the colon or the rectum. It is the third most common cancer type in males and the second most common cancer type in females (1). CRC also accounts for $\sim 10 \%$ of annual global cancer incidence, which is increasing with economic development (2). Furthermore, the 5-year survival rate decreases at lower levels of income, with rates reaching $60 \%$ in high-income countries but falling to $30 \%$ or less in low-income countries $(3,4)$.

Correspondence to: Professor Feng Qiu, Department of Laboratory Medicine, Nanhai Hospital, Southern Medical University, 28 Liguan Road, Foshan, Guangdong 528244, P.R. China

E-mail: QFSFL@126.com

Key words: CRMP5, proliferation, migration, MAPK signaling, ERK1/2, colorectal cancer
The low survival rate is due to metastasis of primary CRC to the liver, lungs and other organs (1,3). Despite breakthroughs in clinical research that have identified numerous genetic and protein abnormalities in $\mathrm{CRC}$, it remains the second leading cause of cancer-related mortality (1). The molecular mechanisms underlying CRC tumorigenesis and progression have not yet been elucidated.

Collapsin response mediator proteins (CRMPs), namely CRMP 1-5, are a family of five homologous multifunctional cytosolic phosphoproteins highly expressed in the developing brain $(5,6)$. They are involved in regulating cell migration through interactions with the cytoskeleton and altered expression levels of CRMPs have been detected in several cancer types, including breast, prostate and lung carcinoma (7-10). The most recently identified CRMP, CRMP5, also known as DPYSL5, is widely expressed in regions of neurogenesis in the adult brain $(11,12)$. CRMP5 was identified as a specific marker of high-grade lung neuroendocrine carcinoma, including small cell lung carcinomas and large cell neuroendocrine carcinomas (13). In glioblastoma, CRMP5 was found to control cancer cell proliferation and survival via Notch-dependent signaling (9). However, the role of CRMP5 in CRC is unclear.

The mitogen-activated protein kinase (MAPK) family consists primarily of extracellular-signal-regulated kinase (ERK), c-jun N-terminal kinase (JNK) and p38MAPK (14). Each MAPK signaling pathway contains at least three major components, including mitogen-activated protein 3 kinase (MAPKKK), mitogen-activated protein 2 kinase (MAPKK) and MAPK $(14,15)$. Various extracellular and intracellular stimuli, including genotoxic agents, cytokines, hormones, oxidative stress and cell adhesion, can promote sequential activation of these kinase components which phosphorylate various substrates, including transcription factors and enzymes, to regulate multiple cellular physiological or pathological processes, including inflammation, cell proliferation, differentiation, metabolism, survival and death (15-17). However, MAPK serves a pleiotropic role in cancer, and the associated mechanisms of the MAPK pathway in regulating tumors remain elusive.

The present study demonstrated that high CRMP5 expression is associated with poor survival in 367 patients with 
CRC. CRMP5 regulates CRC cell proliferation and development via MAPK signaling. Additionally, an unexpected role of CRMP5-overexpression in promoting CRC chemotherapy resistance and tumor recurrence was identified. The results of the present study elucidated novel mechanisms of CRMP5-induced CRC cell proliferation and development and provided a potential novel target for CRC treatments.

\section{Materials and methods}

Cell culture and transfection. Human CRC HCT116 and HT29 cell lines were purchased from the Cell Resource Center of Shanghai Institutes for Biological Sciences, Chinese Academy of Sciences. Cell lines were authenticated by STR analysis (Shanghai Biotechnology Co., Ltd., http://www. cellresource.cn/index.aspx). Cells were grown in Dulbecco's minimal essential medium (DMEM, Gibco; Thermo Fisher Scientific, Inc.) supplemented with $10 \%$ fetal bovine serum (FBS; Gibco; Thermo Fisher Scientific, Inc.) and 1\% penicillin streptomycin-glutamine (Gibco; Thermo Fisher Scientific, Inc.) at $37^{\circ} \mathrm{C}$ with $5 \% \mathrm{CO}_{2}$.

Human CRMP5-overexpression or -knockdown plasmids were constructed using $\mathrm{pCDH}$ and $\mathrm{pLKO} .1$ vectors (Genomeditech), respectively. Recombinant plasmids and packaging plasmids, including VSVG $(0.75 \mu \mathrm{g}$, Addgene $)$ and psPAX2 (1.72 $\mu \mathrm{g}$, Addgene), were transiently transfected into 293 cells (Shanghai Zhongqiao Xinzhou Biotechnology Co., Ltd.) to produce lentivirus using Lipofectamine ${ }^{\circledR} 2000$ (Invitrogen; Thermo Fisher Scientific, Inc.), according to the manufacturer's protocol. Cultured CRC cells were infected with the appropriate lentivirus in the presence of polybrene $\left(5 \mu \mathrm{g} / \mathrm{ml}\right.$; Sigma-Aldrich; Merck KGaA) at $37^{\circ} \mathrm{C}$ for $12 \mathrm{~h}$, prior to being selected using puromycin $(2 \mu \mathrm{g} / \mathrm{ml}$; Sigma-Aldrich; Merck KGaA). An empty vector or a nonsense scrambled oligonucleotide was used as a negative control. Following selection with puromycin $(2 \mu \mathrm{g} / \mathrm{ml}$; Sigma-Aldrich; Merck $\mathrm{KGaA}$ ) for 2 week at $37^{\circ} \mathrm{C}$, transfected cells were harvested for subsequent experimentation. CRMP5 gene expression of the transfected cells was evaluated via reverse transcriptionquantitative (RT-q)PCR and western blot analyses.

Western blotting. Cultured CRC cells treated without or with selumetinib $(500 \mathrm{nM})$ at $37^{\circ} \mathrm{C}$ for $24 \mathrm{~h}$ were collected and lysed with 1X RIPA Lysis Buffer (Sigma-Aldrich; Merck KGaA) containing 1X Protease Inhibitor Cocktail (Sigma-Aldrich; Merck KGaA). The concentration of total protein was measured with a BCA protein assay kit (Beyotime Institute of Biotechnology). Total protein (30 $\mu \mathrm{g} / \mathrm{lane}$ ) was separated using 6-12.5\% SDS-PAGE, transferred to a polyvinylidene fluoride membrane, and blocked with 5\% skimmed milk at room temperature for $1 \mathrm{~h}$. Next, membranes were incubated with primary antibodies against CRMP5 (Abcam; cat. no. ab36203; 1:2,000), Ki67 (Abcam; cat. no. ab15580; 1:1,000), Caspase-3 (CST; cat. no. 96659665; 1:1,000), E-cadherin (CST; cat. no. 3195; 1:1,000), MMP-2 (CST; cat. no. 4022; 1:1,000), MMP-9 (CST; cat. no. 13667; 1:1,000) and vimentin (CST; cat. no. $5741 ; 1: 1,000$ ) overnight at $4{ }^{\circ} \mathrm{C}$, followed by incubation with fluorescence-conjugated goat ant-mouse IgG $\mathrm{H} \& \mathrm{~L}$ (Abcam; cat. no. ab216772) or goat an-rabbit IgG H\&L (Abcam; cat. no. ab216773) secondary antibodies (dilution, 1:1,000) at room temperature for $1 \mathrm{~h}$. Finally, bands were detected using a two-color infrared laser imaging system (Odyssey; LI-COR Biosciences).

Cell proliferation assays. For colony formation assay, cells $(1,000 /$ well $)$ were seeded into 12 -well plates and cultured in fresh DMEM medium (Gibco; Thermo Fisher Scientific, Inc.) with or without selumetinib $(500 \mathrm{nM})$ for approximately two weeks to allow colony formation. Cells were then fixed with $4 \%$ paraformaldehyde at room temperature for $10 \mathrm{~min}$, and stained with $1 \%$ crystal violet at room temperature for $20 \mathrm{~min}$. The number of colonies was counted using ImageJ software (v1.51; National Institutes of Health). Cell viability was measured using the Cell Counting kit-8 (CCK-8) assay. Cultured CRC cells $(2,000$ cells/well) were seeded into 96-well plates for $24 \mathrm{~h}$. The next day, cells were treated with increasing doses $(0,1,5,10,50,100$ and $200 \mu \mathrm{M})$ of 5-FU (Sigma-Aldrich; Merck KGaA) at $37^{\circ} \mathrm{C}$ for $48 \mathrm{~h}$. Next, CCK-8 solution (10 $\mu \mathrm{l} /$ well; Dojindo Molecular Technologies, Inc.) was added to each well and absorbance was measured at $450 \mathrm{~nm}$ using a microplate reader.

Transwell migration assay. CRC cells $\left(2 \times 10^{5}\right.$ cells/well $)$ in $200 \mu 1$ complete DMEM medium (Gibco; Thermo Fisher Scientific, Inc.) were seeded into the upper Transwell chamber (12- $\mu \mathrm{M}$ pore size; BD Biosciences) and DMEM medium (Gibco; Thermo Fisher Scientific, Inc.) with 15\% FBS was plated into the lower chamber. After $48 \mathrm{~h}$ at $37^{\circ} \mathrm{C}$ in culture, cells adhering to the underside of the transmembrane were fixed with $20 \%$ methanol for $10 \mathrm{~min}$ at room temperature, and then stained with $0.1 \%$ crystal violet for $1 \mathrm{~h}$ at room temperature. Finally, the images were captured using an inverted microscope (Carl Zeiss AG; magnification, x100).

$R T-q P C R$. Total RNA was extracted from cells using the RNeasy Mini kit (Qiagen $\mathrm{GmbH}$ ) and reverse transcribed into cDNA with specific oligo (dT) primers using the Prime Script RT Reagent kit (Takara Bio, Inc.). The temperature and duration of RT were: $37^{\circ} \mathrm{C}$ for $15 \mathrm{~min}$, followed by $85^{\circ} \mathrm{C}$ for 5 sec. RT-qPCR was performed in 96-well plates using SYBR Premix Ex Taq II kit (Takara Biotechnology Co., Ltd.), on an Applied Biosystems 7300 Real-Time PCR System (Thermo Fisher Scientific, Inc.) to measure mRNA levels. The thermocycling conditions used were as follows: $95^{\circ} \mathrm{C}$ for $30 \mathrm{sec}$, followed by 40 cycles of $95^{\circ} \mathrm{C}$ for $10 \mathrm{sec}$ and $60^{\circ} \mathrm{C}$ for $30 \mathrm{sec}$. The $2^{-\Delta \Delta \mathrm{Ca}}$ method (18) was used to calculate relative mRNA levels and GAPDH was used for normalization of specific mRNA expression. Primers used in RT-qPCR are listed in Table SI.

The Cancer Genome Atlas (TCGA) data analysis. The Colorectal adenocarcinoma dataset (dataset no. TCGACOADREAD) from TCGA (https://portal.gdc.cancer.gov) was downloaded to determine the correlation between selected gene (DPYSL5) and patient survival $(19,20)$. A total of 376 patients with transcriptional profiles and basic clinical information, including age, sex, stage, overall survival and survival status were enrolled in the present study. RNaseq v2 mRNA expression data and clinical information were retrieved from the cBioportal (21). Survival analysis was performed using 
the R package survival (v3.5.3; http://www.bioconductor.org/ packages), and survival curves were fit using the survfit function. The difference between high- and low-expression groups was tested by survdiff using the median as the cut-off.

RNA-sequencing. RNA was extracted using Direct-zol ${ }^{\text {TM }}$ RNA kit (Zymo Research Corp.), purified with RNeasy Plus Mini kit (Qiagen $\mathrm{GmbH}$ ). One hundred nanograms of mRNA was used as input to the TruSeq mRNA Sample Prep v2 kit (Illumina, Inc.), with the poly-A pulldown step. Sample preparation was performed according to the manufacturer's protocols. The libraries were sequenced on HiSeq $X$ ten (Illumina, Inc.) using $150 \mathrm{bp}$ paired-end reads. The sequencing data termed raw data (raw reads) were subjected to quality control (QC). Following QC, raw data were filtered into clean data, and clean reads were aligned to the reference genome (February 2009, GRCh37/hg19) using BWA (v0.7.12). Genes with an adjusted P-value of $<0.05$ identified by DESeq2 (v1.12.3) were defined as differentially expressed. The statistical enrichment of differentially expressed genes in Kyoto Encyclopedia of Genes and Genomes (KEGG) pathways analysis was determined by Cluster Profiler (v3.0.4).

Statistical analysis. Data are presented as the mean \pm standard deviation of three independent experiments. Student's t-test or one-way analysis of variance, followed by the Bonferroni post hoc test, was used to compare the treated and corresponding control groups. Survival curves were estimated using the Kaplan-Meier method and analyzed by the log-rank test. $\mathrm{P}<0.05$ was considered to indicate a statistically significant difference.

\section{Results}

High CRMP5 expression is associated with poor survival in TCGA patients with colorectal cancer. To evaluate the clinical significance of CRMP5 in CRC, survival analysis was conducted using TCGA COADREAD data. Notably, high expression of CRMP5 was significantly associated with poor survival in 367 patients from COADREAD (Fig. 1A). Furthermore, co-expression analysis by Pearson correlation demonstrated that the majority of genes co-expressed with CRMP5 were mainly associated with the immune response (Fig. 1B and C). These data suggested that CRMP5 may serve an important role in tumor inflammation, thereby promoting cancer development and subsequently causing poor survival.

CRMP5-overexpression promotes cell proliferation and development of $C R C$. To investigate the potential role of CRMP5 in CRC, CRMP5 was overexpressed in HT29 cells using lentivirus-mediated human CRMP5 cDNA. CRMP5-overexpression was confirmed by RT-qPCR and Western blotting (Fig. 2A). HT29 cells overexpressing CRMP5 had an increase in cell number compared with the wild-type (WT) cells and cells transfected with empty plasmid (NC), indicating that high levels of CRMP5 promoted CRC cell proliferation (Fig. 2B). This result was further confirmed by a colony formation assay which demonstrated that CRMP5-overexpression significantly increased the colony forming ability of HT29 cells (Fig. 2C). Additionally, Transwell migration assays demonstrated that CRMP5-overexpression promoted HT29 cell migration (Fig. 2D). Additionally, the expression levels of common markers for cell proliferation, migration and invasion were measured. As expected, an increase in the expression of $\mathrm{Ki} 67$, a common marker for cellular proliferation, was observed and commonly used markers of migration and invasion, including E-cadherin, vimentin, MMP-2 and MMP-9, while expression of caspase-3, an apoptosis-related protein, was decreased in CRMP5-OE HT29 cells compared with WT or NC as detected by RT-qPCR and Western blotting (Fig. 2E and F).

CRMP5-knockdown inhibits cell proliferation and development of $C R C$. CRMP5 expression was downregulated in HCT116 cells using shRNA-encoding lentiviruses. RT-qPCR and Western blotting confirmed CRMP5-knockdown in HCT116 cells (Fig. 3A). Additionally, the results of the present study demonstrated that CRMP5-knockdown markedly inhibited cell proliferation and migration (Fig. 3B-D). Similarly, CRMP5-knockdown also increased caspase-3 expression and decreased the expression of Ki67, E-cadherin, vimentin, MMP-2 and MMP-9 (Fig. 3E and F). Taken together, these data indicated that CRMP5 regulates CRC cell proliferation and serves a critical role in regulating CRC development.

MAPK signaling is involved in regulating CRMP5-induced $C R C$ cell proliferation and tumor recurrence. To further investigate the molecular mechanisms of CRMP5 in regulating CRC development, RNA-Seq analysis was performed in CRMP5-overexpressed and -knockdown cell lines. KEGG pathway enrichment analysis demonstrated that differentially expressed genes in CRMP5-overexpression or -knockdown cells were significantly enriched in the MAPK and metabolic signaling pathways (Fig. 4A and B). To further investigate the regulatory mechanisms of CRMP5 in CRC, MAPK signaling was selectively blocked by using Selumetinib (AZD6244), a potent, selective and ATP-uncompetitive inhibitor of MAPK/ERK1/2 to determine their effect on cell proliferation. Notably, it was observed that inhibiting ERK1/2 activity with selumetinib markedly alleviated the change in colony formation, as well as the changes in Ki67, E-cadherin, vimentin, MMP-2, MMP-9 and caspase-3 expression levels in HCT116 and HT29 cell lines induced by CRMP5 (Fig. 4C and D). These data suggested that CRMP5 may regulate CRC cell proliferation and development through MAPK signaling.

Overexpression of CRMP5-induced chemotherapy resistance and tumor recurrence of CRC. Therefore, the role of CRMP5 in chemotherapeutic drug resistance was examined. CRMP5-overexpression and control CRC cells were treated with increasing doses of 5-fluorouracil (5-FU), a chemotherapeutic drug commonly used in CRC (22). Results of CCK-8 assays demonstrated that 5-FU treatment significantly decreased the viability of CRC cells while CRMP5 overexpression significantly reversed the inhibitory effects induced by the drug (Fig. 5A). Additionally, a retrospective study was performed using 220 clinical samples from patients with CRC in a well-established TCGA-COAD cohort to determine the role of CRMP5 in CRC recurrence. The results of the present study demonstrated that high expression of CRMP5 
A

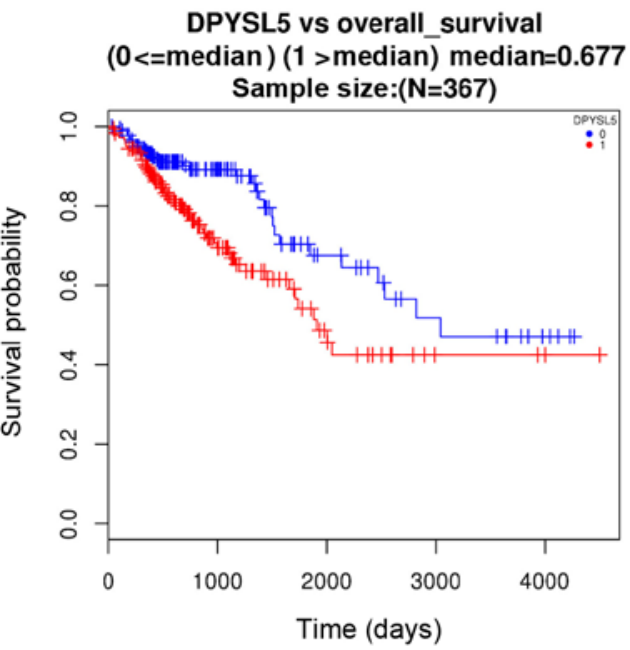

C
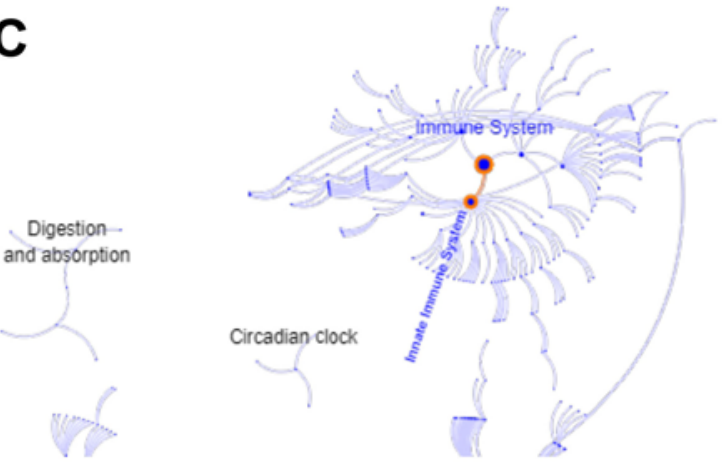

B

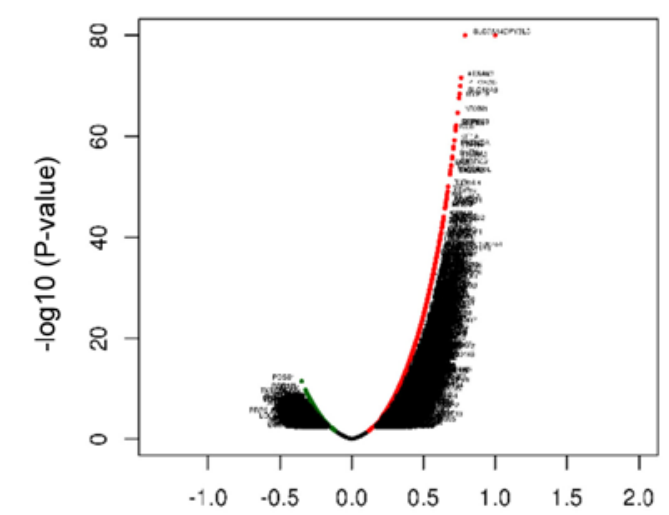

Pearson correlation coefficient (Pearson test)

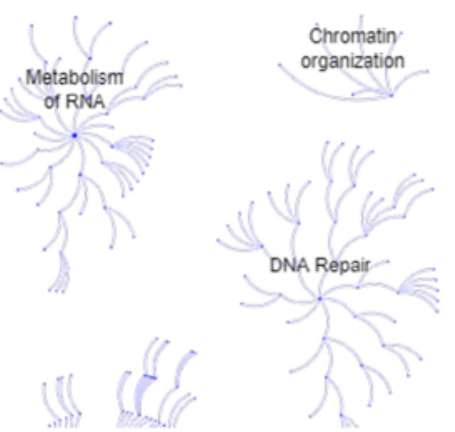

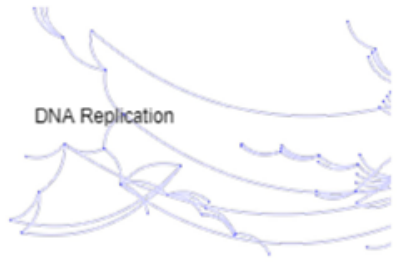

Muscle contraction

Figure 1. Clinical significance of CRMP5 (DPYSL5) in CRC from TCGA data. (A) Overall survival of patients with CRC in CRMP5 (DPYSL5) high- and low-expression groups $(\mathrm{P}=0.016)$. (B) Genes co-expressed with CRMP5 (DPYSL5) analyzed by Pearson correlation. (C) Gene function and pathway enrichment of CRMP5 (DPYSL5) co-expressed genes. CRMP5, Collapsin response mediator protein 5; CRC, colorectal cancer; TCGA, the Cancer Genome Atlas.

A

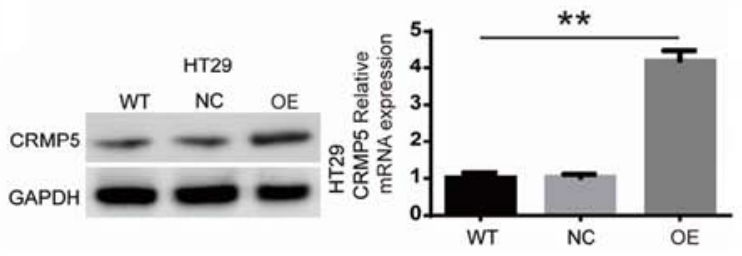

C

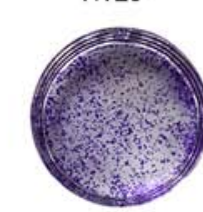

HT29-NC

HT29-OE

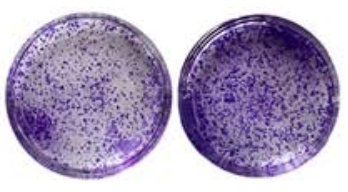

D

HT29

HT29-NC

HT29-OE

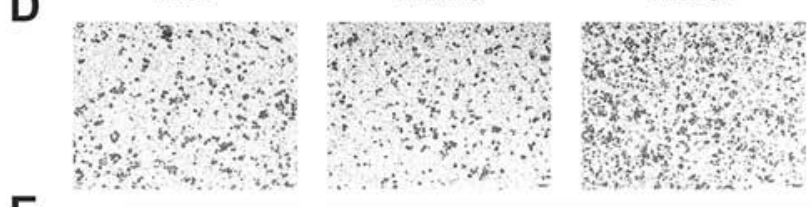

E
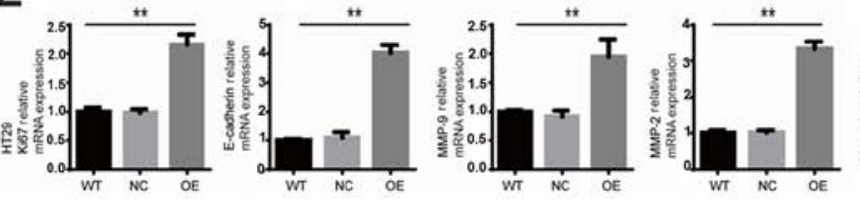

B
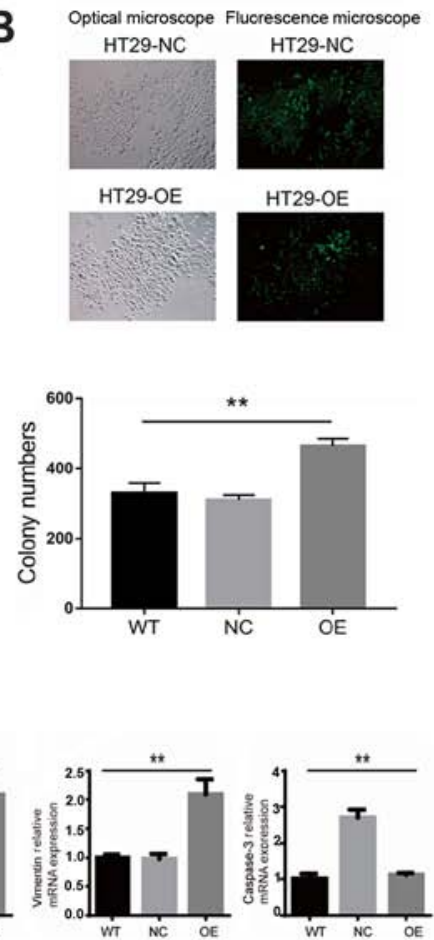

F
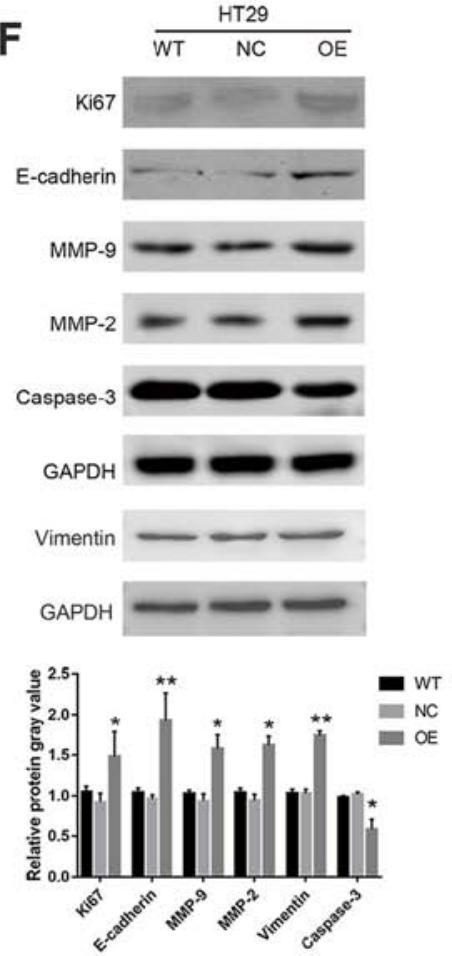

Figure 2. CRMP5-overexpression promotes proliferation of HT29 cells. (A) Western blotting and qPCR analysis of CRMP5 expression levels. (B) Optical and fluorescence microscopy of HT29 cells. (C) Colony number and (D) cell migration of HT29 WT, NC and CRMP5 OE cells. (E) qPCR and (F) Western blotting analysis of Ki67, E-cadherin, vimentin, MMP-2, MMP-9 and caspase-3 expression levels. All experiments were repeated at least three times. Data are presented as the mean \pm standard deviation, ${ }^{*} \mathrm{P}<0.05,{ }^{* *} \mathrm{P}<0.01$. CRMP5, Collapsin response mediator protein 5; qPCR, quantitative polymerase chain reaction; WT, wild-type; NC, negative control; OE, overexpression. 
A
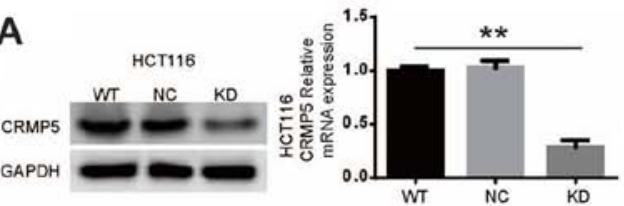

HCT116 HCT116-NC HCT116-KD

C
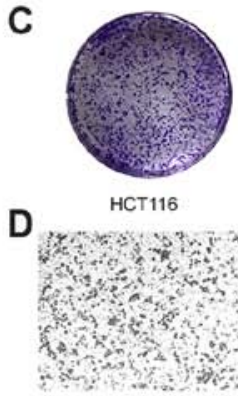

E

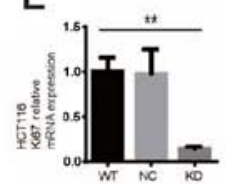

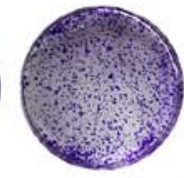

HCT116-NC
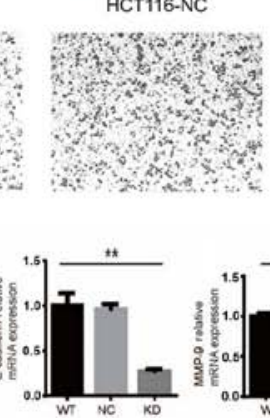

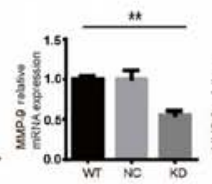

HCT116-KD

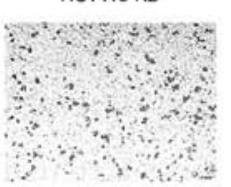

B
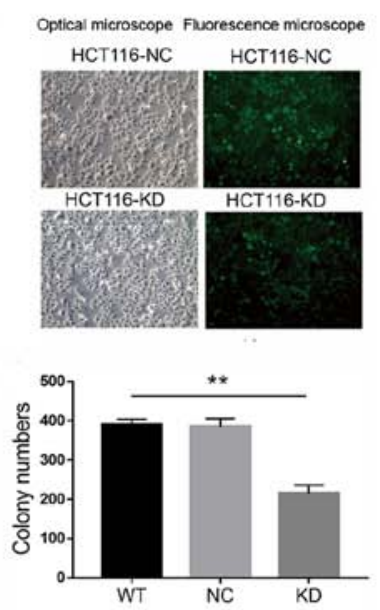

Figure 3. CRMP5-KD inhibits proliferation of HCT116 cells. (A) Western blotting and qPCR analysis of CRMP5 expression levels. (B) Optical and fluorescence microscopy of the HCT116 cells. (C) Colony number and (D) cell migration of HCT116 WT, NC and CRMP5-KD cells. (E) qPCR and (F) Western blot analysis of Ki67, E-cadherin, vimentin, MMP-2, MMP-9 and caspase-3 expression levels. All experiments were repeated at least three times. Data are presented as the mean \pm standard deviation, ${ }^{*} \mathrm{P}<0.05,{ }^{* *} \mathrm{P}<0.01,{ }^{* * *} \mathrm{P}<0.001$. CRMP5, Collapsin response mediator protein 5; qPCR, quantitative polymerase chain reaction; WT, wild-type; $\mathrm{NC}$, negative control; $\mathrm{KD}$, knockdown.

A

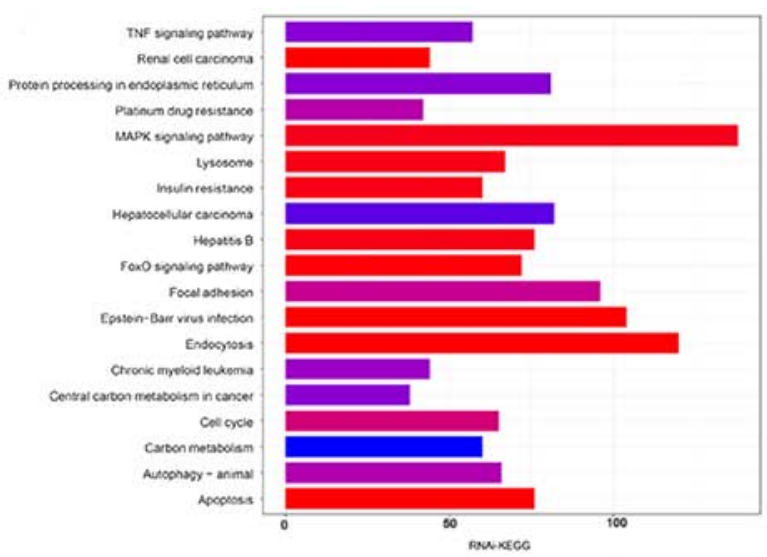

$\mathrm{C}$

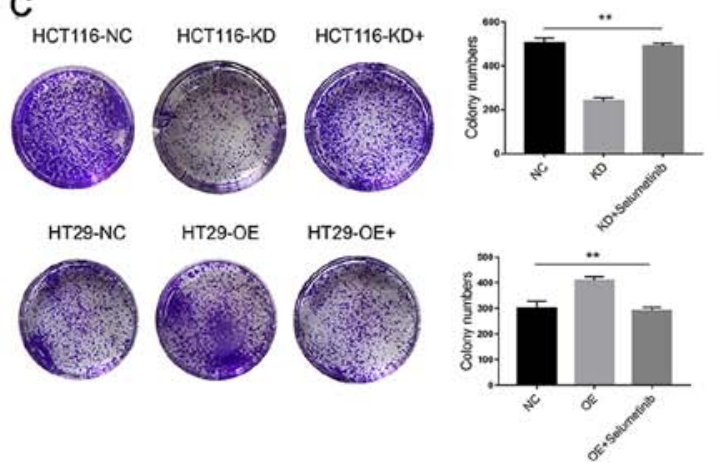

B

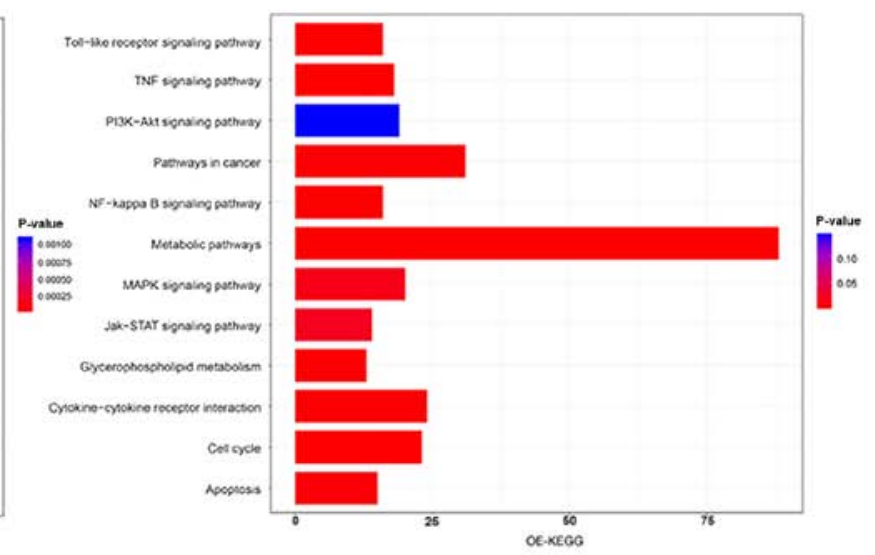

D

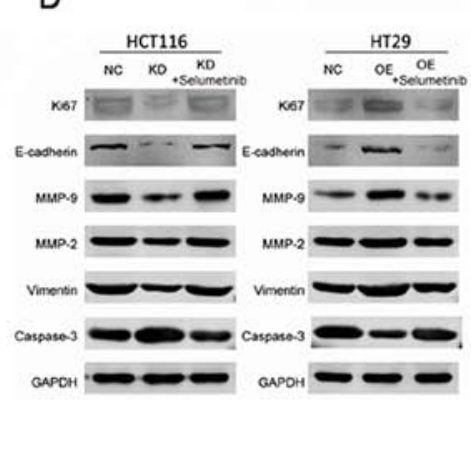

F
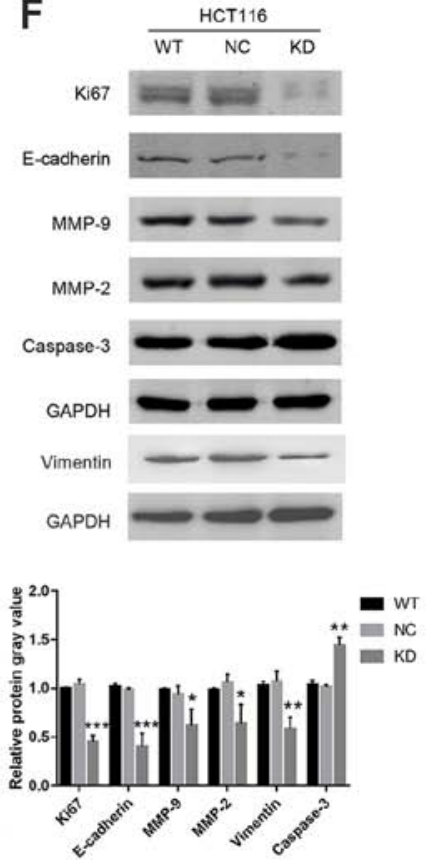
A

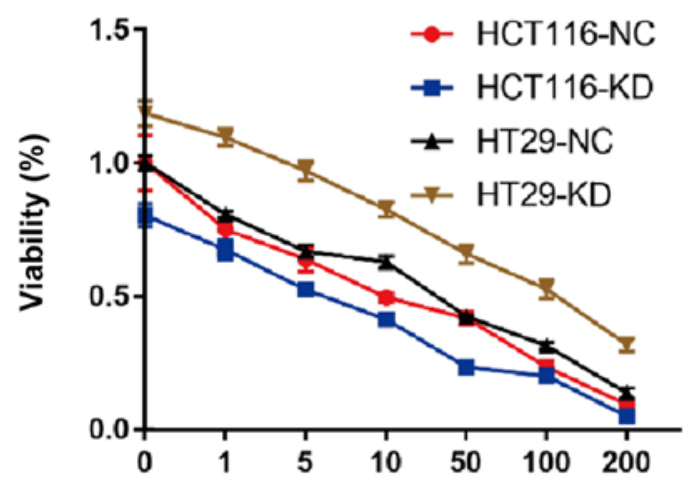

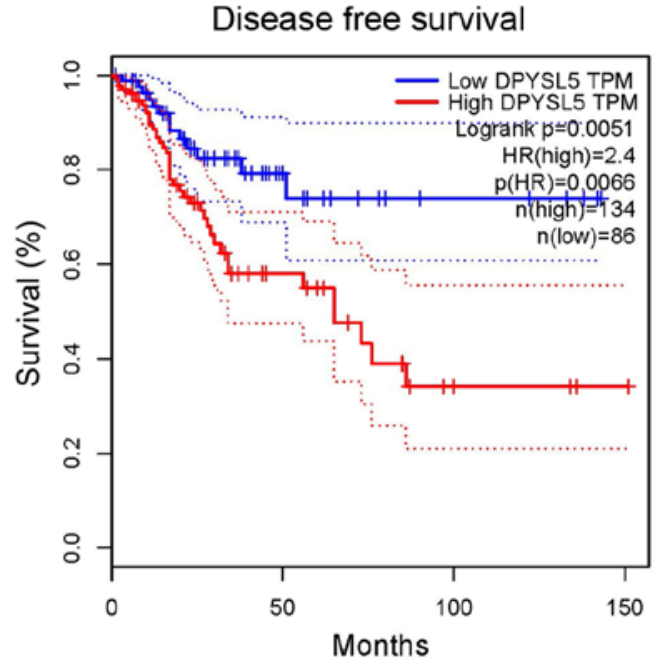

Figure 5. CRMP5-overexpression induces chemotherapy resistance and tumor recurrence of CRC. (A) Chemotherapy drug sensitivity of CRMP5 overexpression cells and corresponding NC cells. (B) Disease-free survival analysis of CRMP5 (DPYSL5) using 220 clinical samples from CRC patients in TCGA-COAD cohort. The level of CRMP5 is defined by the median and the dotted lines on the KM plot represent 95\% confidence intervals. CRMP5, Collapsin response mediator protein 5; CRC, colorectal cancer; NC, negative control; KD, knockdown.

significantly indicated poor disease-free survival of patients with CRC (Fig. 5B). Taken together, these data suggested that CRMP5-overexpression induces chemotherapy resistance and tumor recurrence of CRC.

\section{Discussion}

$\mathrm{CRC}$ is one of the most frequent malignancies worldwide with incidence and mortality rates still rising rapidly in many low-income and middle-income countries (3). Combination therapies, including surgery, local radiotherapy and chemotherapy, are effective against the early stages of CRC. However, resistance to traditional chemotherapeutic drugs is frequently observed in patients with advanced stages of disease, eventually resulting in the development and relapse of tumors $(22,23)$. Therefore, the identification of novel biomarkers involved in CRC development and recurrence has potential applications in anticancer treatment.

Altered CRMP expression levels have been detected in multiple cancer types, and several CRMP proteins have been reported to be upregulated in certain cancer types and contribute toward cancer progression and metastasis (7-10). As a member of the CRMP family, CRMP5 antibodies have been demonstrated to be associated with thymoma or small-cell lung cancer, suggesting that CRMP5 may be involved in these malignancies $(24,25)$. A previous inter-laboratory study revealed that strong and extensive CRMP5 expression was observed in $98.6 \%$ of high-grade neuroendocrine lung tumors (13). These results suggested that CRMP5 may be a useful marker for routine pathological evaluation of lung tumor surgical samples to distinguish between highly aggressive neuroendocrine carcinoma and other lung cancers (13). Another study demonstrated that CRMP5 was an indicator of poor survival and promoted cell proliferation of glioblastoma (9). A recent study also reported that CRMP5 promoted osteosarcoma cell growth, and high expression levels of DRP5 were associated with a poor prognosis in patients with osteosarcoma (26). To the best of our knowledge, the present study was the first to demonstrate that CRMP5-overexpression was significantly associated with poor survival in patients with CRC. Cell line experiments demonstrated that CRMP5-overexpression promoted cell proliferation and migration, while CPMP5-knockdown in CRC cells decreased cell growth and migration. Additionally, it was found that CRMP5-overexpression induced CRC recurrence and chemotherapy resistance to 5-FU, a commonly used chemotherapeutic drug in CRC. The results suggested that CPMP5 may be used as a potential novel therapeutic target for CRC. However, further validation and analysis of the effects of CPMP5 in related in vivo animal models are required.

Members of the MAPK family, including ERK, JNK and p38MAPK, are involved in regulating cell proliferation, survival and chemoresistance of multiple cancer types, including CRC $(14,16)$. The RNA-Seq and KEGG pathway enrichment analysis revealed that differentially expressed genes following CRMP5-overexpression or -knockdown were significantly enriched in the MAPK signaling pathway. In addition, it was found that inhibiting ERK1/2 markedly alleviated the changes in migration and invasion induced by CRMP5 in CT116 and HT29 cells. A previous study on glioblastoma revealed that increased CRMP5 levels promoted Notch receptor expression and Akt activation in human tumor cell lines (9). Additionally, CRMP5 functions by hijacking Notch receptors from Itch-dependent lysosomal degradation (9). Our findings suggested that MAPK signaling is involved in regulating CRMP5-induced CRC cell proliferation and development, suggesting that MAPK signaling inhibition has the potential to be utilized in CRC treatment.

In summary, CRMP5 was characterized as a novel biomarker of poor survival in CRC that regulates tumor cell proliferation and development. The present study uncovered a novel mechanism by which CRMP5 regulates the MAPK signaling pathway, to drive CRC cell proliferation and development. The results of the present study suggested that CRMP5 may be a novel therapeutic target for CRC. 


\section{Acknowledgements}

Not applicable.

\section{Funding}

The present study was supported by grants from the National Natural Science Foundation of China (grant no. 81503424), Guangzhou Science and Technology Innovation Commission (grant no. 201704020171), the Medical Key Discipline Planning Project of Foshan City (grant no. FSZDZK135045), and the Science and Technology Planning Project of Guangdong Province (grant no. 2019B020208009).

\section{Availability of data and materials}

The datasets used and/or analyzed during the current study are available from the corresponding author upon reasonable request.

\section{Authors' contributions}

FHQ and FQ designed the experiments, analyzed the data and drafted the initial manuscript. FHQ and FQ confirmed the authenticity of all the raw data. FHQ, LY, DL and YW performed the experiments. All authors discussed the results and approved the final version of the manuscript.

\section{Ethics approval and consent to participate}

Not applicable.

\section{Patient consent for publication}

Not applicable.

\section{Competing interests}

The authors declare that they have no competing interests.

\section{References}

1. El-Shami K, Oeffinger KC, Erb NL, Willis A, Bretsch JK, Pratt-Chapman ML, Cannady RS, Wong SL, Rose J, Barbour AL, et al: American cancer society colorectal cancer survivorship care guidelines. CA Cancer J Clin 65: 428-455, 2015.

2. Keum N and Giovannucci E: Global burden of colorectal cancer: Emerging trends, risk factors and prevention strategies. Nat Rev Gastroenterol Hepatol 16: 713-732, 2019.

3. Arnold M, Sierra MS, Laversanne M, Soerjomataram I, Jemal A and Bray F: Global patterns and trends in colorectal cancer incidence and mortality. Gut 66: 683-691, 2017.

4. Ferlay J, Colombet M, Soerjomataram I, Dyba T, Randi G, Bettio M, Gavin A, Visser O and Bray F: Cancer incidence and mortality patterns in Europe: Estimates for 40 countries and 25 major cancers in 2018. Eur J Cancer 103: 356-387, 2018.

5. Charrier E, Reibel S, Rogemond V, Aguera M, Thomasset N and Honnorat $\mathrm{J}$ : Collapsin response mediator proteins (CRMPs): Involvement in nervous system development and adult neurodegenerative disorders. Mol Neurobiol 28: 51-64, 2003

6. Quach TT, Honnorat J, Kolattukudy PE, Khanna R and Duchemin AM: CRMPs: Critical molecules for neurite morphogenesis and neuropsychiatric diseases. Mol Psychiatry 20 $1037-1045,2015$
7. Tan F, Thiele CJ and Li Z: Collapsin response mediator proteins: Potential diagnostic and prognostic biomarkers in cancers (Review). Oncol Lett 7: 1333-1340, 2014.

8. Gao X, Mao YH, Xiao C, Li K, Liu W, Li LY and Pang J: Calpain-2 triggers prostate cancer metastasis via enhancing CRMP4 promoter methylation through NF-kappaB/DNMT1 signaling pathway. Prostate 78: 682-690, 2018.

9. Moutal A, Honnorat J, Massoma P, Désormeaux P, Bertrand C, Malleval C, Watrin C, Chounlamountri N, Mayeur ME, Besançon R, et al: CRMP5 controls glioblastoma cell proliferation and survival through notch-dependent signaling. Cancer Res 75: 3519-3528, 2015.

10. Gozzard P, Woodhall M, Chapman C, Nibber A, Waters P, Vincent A, Lang B and Maddison P: Paraneoplastic neurologic disorders in small cell lung carcinoma: A prospective study. Neurology 85: 235-239, 2015.

11. Veyrac A, Reibel S, Sacquet J, Mutin M, Camdessanche JP, Kolattukudy P, Honnorat J and Jourdan F: CRMP5 regulates generation and survival of newborn neurons in olfactory and hippocampal neurogenic areas of the adult mouse brain. PLoS One 6: e23721, 2011.

12. Ricard D, Rogemond V, Charrier E, Aguera M, Bagnard D, Belin MF, Thomasset $\mathrm{N}$ and Honnorat $\mathrm{J}$ : Isolation and expression pattern of human Unc-33-like phosphoprotein 6/collapsin response mediator protein 5 (Ulip6/CRMP5): Coexistence with Ulip2/CRMP2 in Sema3a- sensitive oligodendrocytes. J Neurosci 21: 7203-7214, 2001.

13. Meyronet D, Massoma P, Thivolet F, Chalabreysse L, Rogemond V, Schlama A, Honnorat J and Thomasset N: Extensive expression of collapsin response mediator protein 5 (CRMP5) is a specific marker of high-grade lung neuroendocrine carcinoma. Am J Surg Pathol 32: 1699-1708, 2008.

14. Morrison DK: MAP kinase pathways. Cold Spring Harb Perspect Biol 4: a011254, 2012

15. Su B and Karin M: Mitogen-activated protein kinase cascades and regulation of gene expression. Curr Opin Immunol 8: 402-411, 1996.

16. Dhillon AS, Hagan S, Rath O and Kolch W: MAP kinase signalling pathways in cancer. Oncogene 26: 3279-3290, 2007.

17. Burotto M, Chiou VL, Lee JM and Kohn EC: The MAPK pathway across different malignancies: A new perspective. Cancer 120: 3446-3456, 2014.

18. Livak KJ and Schmittgen TD: Analysis of relative gene expression data using real-time quantitative PCR and the 2(-Delta Delta C(T)) method. Methods 25: 402-408, 2001

19. Lee JS: Exploring cancer genomic data from the cancer genome atlas project. BMB Rep 49: 607-611, 2016.

20. Seshagiri S, Stawiski EW, Durinck S, Modrusan Z, Storm EE, Conboy CB, Chaudhuri S, Guan Y, Janakiraman V, Jaiswal BS, et al: Recurrent R-spondin fusions in colon cancer. Nature 488: 660-4, 2012.

21. Cerami E, Gao J, Dogrusoz U, Gross BE, Sumer SO, Aksoy BA, Jacobsen A, Byrne CJ, Heuer ML, Larsson E, et al: The cBio cancer genomics portal: An open platform for exploring multidimensional cancer genomics data. Cancer Discov 2: 401-404, 2012.22.

22. Vodenkova S, Buchler T, Cervena K, Veskrnova V, Vodicka P and Vymetalkova V: 5-fluorouracil and other fluoropyrimidines in colorectal cancer: Past, present and future. Pharmacol Ther 206: 107447, 2019.

23. Hammond WA, Swaika A and Mody K: Pharmacologic resistance in colorectal cancer: A review. Ther Adv Med Oncol 8: 57-84, 2016.

24. Monstad SE, Nostbakken JK and Vedeler CA: CRMP5 antibodies found in a patient with limbic encephalitis and myasthenia gravis. J Neurol Neurosurg Psychiatry 80: 241-242, 2009.

25. Monstad SE, Drivsholm L, Skeie GO, Aarseth JH and Vedeler CA: CRMP5 antibodies in patients with small-cell lung cancer or thymoma. Cancer Immunol Immunother 57: 227-232, 2008.

26. Wang L, Liu W, Tang H, Xie X, Zou C, Wang Y, Gao Z and Yin J: DRP5 is involved in cancer cell growth and predicts poor prognosis in human osteosarcoma. Cancer Med 6: 982-993, 2017.

(i) (2) This work is licensed under a Creative Commons Attribution-NonCommercial-NoDerivatives 4.0 International (CC BY-NC-ND 4.0) License. 